\title{
Takotsubo cardiomyopathy and microcirculatory dysfunction
}

Nauman Khalid and Lovely Chhabra

We read with great interest the Review by Akashi et al. (Epidemiology and pathophysiology of Takotsubo syndrome. Nat. Rev. Cardiol. doi:10.1038/nrcardio.2015.39), ${ }^{1}$ which provides a comprehensive summary of the underlying pathogenesis of Takotsubo cardiomyopathy. However, we wish to highlight a few important points relevant to the article.

Thrombolysis In Myocardial Infarction (TIMI) frame count (TFC) has been used as a quantitative index to determine microcirculatory function in patients with Takotsubo cardiomyopathy. ${ }^{2-5}$ Defined as 'the number of frames required for contrast material to travel from the coronary ostium to the standardized distal landmark', TFC is a simple yet inexpensive test for calculation of the coronary flow reserve. ${ }^{6}$ The term ' corrected TIMI frame count' (CTFC) is applied when a correction factor is used to compensate for the longer length of the left anterior descending coronary artery (LAD) compared with the circumflex and right coronary arteries. ${ }^{6}$

We conducted a retrospective study to explore microcirculatory analysis in patients with Takotsubo cardiomyopathy by calculating TFC in the three main coronary vessels: the $\mathrm{LAD}$, circumflex artery, and right coronary artery., ${ }^{2,3}$ Our results showed that CTFC was significantly higher in the LAD of patients with Takotsubo cardiomyopathy than in the LAD of control individuals, with no difference observed between the two groups in other vessels. Important implications of our study were: patients with Takotsubo cardiomyopathy have an abnormal CTFC in the LAD territory suggesting abnormalities in endothelial or microvascular function, and CTFC disturbances in that particular anatomical location might credibly explain why the apex of the heart is invariably affected in Takotsubo cardiomyopathy, whereas the base of the heart is usually spared.

Some other studies support our findings. Bybee et al. also demonstrated abnormal TFC in all three coronary vessels (not only the LAD) in the acute phase of Takotsubo cardiomyopathy, suggesting diffuse impairment of coronary microcirculatory function. ${ }^{4}$ Kurisu et al. showed similar results in both the acute phase and follow-up. ${ }^{5}$ These studies show that TFC can be an index of myocardial perfusion and a useful marker in patients with Takotsubo cardiomyopathy.

Takotsubo cardiomyopathy is a fascinating illness with ever-growing research to explore its underlying pathophysiology. Microcirculatory dysfunction (especially in the acute phase) has a pivotal role in the evolution of this cardiac syndrome. TFC might be an important diagnostic marker for assessing endothelial or microvascular dysfunction in these patients.

Department of Cardiovascular Medicine, University of Connecticut Health Center, 263 Farmington Avenue, Farmington, CT 06030, USA (N.K.). Hartford Hospital, 80 Seymour Street, Hartford, CT 06102, USA (L.C.).

Correspondence to: N.K.

naumankhalid84@hotmail.com

\section{Competing interests}

The authors declare no competing interests.

1. Akashi, Y. J., Nef, H. M. \& Lyon, A. R. Epidemiology and pathophysiology of Takotsubo syndrome. Nat. Rev. Cardiol. http:// dx.doi.org/10.1038/nrcardio.2015.39.

2. Khalid, N. et al. Thrombolysis In Myocardial Infarction frame count in Takotsubo cardiomyopathy. Int. J. Cardiol. 191, 107-108 (2015).

3. Khalid, N., Iqbal, I. \& Ikram, I. Thrombolysis In Myocardial Infarction frame count in Takotsubo cardiomyopathy [abstract]. J. Am. Coll. Cardiol. 61 (Suppl.), E50 (2013).

4. Bybee, K. A. et al. Systematic review: transient left ventricular apical ballooning: a syndrome that mimics ST-segment elevation myocardial infarction. Ann. Intern. Med. 141, 858-865 (2004).

5. Kurisu, S. et al. Myocardial perfusion and fatty acid metabolism in patients with Tako-tsubolike left ventricular dysfunction. J. Am. Coll. Cardiol. 41, 743-748 (2003).

6. Gibson, C. M. et al. TIMI frame count: a quantitative method of assessing coronary artery flow. Circulation 93, 879-988 (1996). 